\title{
CHARACTERISTIC STUDY OF MHD PUMP WITH CHANNEL IN RECTANGULAR DUCTS
}

Je-Ee Ho

Department of Mechanical Engineering, National Ilan University, Ilan, Taiwan, R.O.C., jeho@niu.edu.tw

Follow this and additional works at: https://jmstt.ntou.edu.tw/journal

Part of the Engineering Commons

\section{Recommended Citation}

Ho, Je-Ee (2007) "CHARACTERISTIC STUDY OF MHD PUMP WITH CHANNEL IN RECTANGULAR DUCTS," Journal of Marine Science and Technology. Vol. 15: Iss. 4, Article 6.

DOI: $10.51400 / 2709-6998.2048$

Available at: https://jmstt.ntou.edu.tw/journal/vol15/iss4/6

This Research Article is brought to you for free and open access by Journal of Marine Science and Technology. It has been accepted for inclusion in Journal of Marine Science and Technology by an authorized editor of Journal of Marine Science and Technology. 


\title{
CHARACTERISTIC STUDY OF MHD PUMP WITH CHANNEL IN RECTANGULAR DUCTS
}

\author{
Je-Ee Ho*
}

Key word: MHD pump, aspect ratio, flow resistance factor.

\begin{abstract}
This study is focused on the prediction of pumping performance in MHD flow. To reach it, an analytic model based on the steady state, incompressible and fully developed laminar flow theory will be provided to analyze the flow characteristic with different scalar dimensions in duct channel. By converting the Lorentz forces which is interacted by electric current and magnetic flux into hydrostatic pressure gradient in the moment equations, the governing equations can be transformed into Poisson equation which makes the analytic solution possible. Furthermore, the concept of flow circuit which is similar to the thermal network in conduction problem will be also proposed in this study.

When compared with experimental results, an excellent agreement was kept among the individual behavior and an easier way to solve the problem could be formulated through the set up of flow circuit.
\end{abstract}

\section{INTRODUCTION}

MHD (Magneto hydrodynamics) has become a very important branch in micro-fluidics for past ten years. The description in fundamental MHD theory is the interaction between the electrically conducting fluid, electric field and magnetic field in the flow channel. When a conducting fluid flows through the device which is equipped with the electric field and magnetic field, working current induced by the electric field will interact with the magnetic field to produce a body force (Lorentz force) as the driving source in the flow.

Though the basic MHD principle has been discussed for years, the study about practical_application was less mentioned before. Jang and Lee [5] published a paper about MHD micro pumps in which the performances of pumps were evaluated by measuring the pressure head under the input of voltage $10-60 \mathrm{~V}$ and magnetic flux density 0.19-0.44 T. Huang et al. [4]

Paper Submitted 09/18/06, Accepted 12/21/06. Author for Correspondence: Je-EeHo.E-mail: jeho@niu.edu.tw

*Department of Mechanical Engineering, National Ilan University, Ilan, Taiwan, R.O.C. demonstrated the pumping characteristic with several aqueous solutions and discussed the pumping efficiency with bubble's formation at the electrode. Geng, et al. [1] predicted the static head of the vapor pressure in the bubble and investigated the application of micro pump in the practical operation. Verardi et al. [6] provided a finite element method to simulate the MHD duct flow through penalty function. With their analysis, an important dimensionless Hartman Number was introduced in the 3-D Navier stroke's equations and the simulated results showed that axial velocity profiles were distorted into $\mathrm{M}$-shape as the magnetic field applied. Wang et al. [7] proposed a simplified MHD flow based on steady state, incompressible and fully developed 2-D laminar flow by taking account of the friction effects on the channel sidewall while the electric field, magnetic field were kept constant. Ho [2, 3] described a static balance between the Lorentz force, body force and surface tension of liquid by measuring the hydraulics head with the applied voltage. From his experiment result, a relevant conclusion told that the effect of bubbles would influence the maximum hydraulics head and low down $50 \%$ peak value approximately.

To build up a flow circuit with analytic solution which was seldom mentioned before, several reasonable assumptions without losing the overall behavior were necessary in this study. Meantime; two important parameter X (aspect ratio), Y (average velocity factor) could be classified from the analytic solution. Through the formulation of function $(\mathrm{X}, \mathrm{Y})$ corresponding to the individual physical characteristic, the required performance would be well discussed respectively and the correct of model was also verified by the experiment test to measure flow rate with various width in duct channel.

\section{FUNDAMENTAL ANALYSIS}

\section{Analytic method}

It is difficult to derive analytic solution by considering all the detail. Several assumptions without causing significant errors will be made as follow. 
(1) Since scalar dimension of the channel in $y$ and $z$ direction are much small compared to that in flow direction $\mathrm{x}$, the flow velocity in $\mathrm{y}$ component, ' $\mathrm{v}$ ' and in $\mathrm{z}$ direction ' $\mathrm{w}$ ' will be assumed as zero by scalar analysis.

(2) As the fully developed case in the flow direction is considered, the axial flow velocity $\mathrm{u}(\mathrm{y}, \mathrm{z})$ is invariant along x direction.

(3) Laminar flow will be presented owing to a small Re number estimated in this study.

(4) The effect of surface tension is neglected because the channel full of the liquid is in use.

(5) Base upon the Ohom's Law, the current density J on the election plate can be expressed as $\vec{J}=\sigma(\vec{E}+\vec{J} \times \vec{B})$. Due to the high conductivity of working fluid is employed, the term of $\mathrm{V} \times \mathrm{B}$ will be dropped out by comparison with E.

(6) Since hydrostatic pressure head in this study just provides the inlet flow velocity for the test channel instead of giving the pressure difference along the channel, the pressure difference primarily caused by the Lorentz force $(\vec{J} \times \vec{B}) L_{p}$ is produced at the region where the length of magnetic $L_{p}$ covers and its effect is taken as uniformly distributed over the entire length

of channel L. That implies $\frac{-\partial p}{\partial x}=\frac{\Delta p}{L}=\frac{\sigma E B L_{p}}{L}$ if the current density vector $\vec{J}$ is perpendicular to the magnetic flux vector $\vec{B}$.

\section{Governing equations}

From above consumptions, the governing equations could be further simplified as:

Continuity equation:

$$
\frac{\partial u}{\partial x}=0
$$

Momentum equations:

$$
0=-\frac{\partial p}{\partial x}+\mu\left(\frac{\partial^{2} u}{\partial y^{2}}+\frac{\partial^{2} u}{\partial z^{2}}\right)
$$

boundary condition:

$$
\begin{aligned}
& x=0, x=L: \frac{\partial u}{\partial x}=0, v=0, \omega=0, \frac{\partial p}{\partial x}=-\frac{\sigma B E L_{p}}{L} \\
& y=-a, y=a: u=0, v=0, \omega=0, \frac{\partial p}{\partial y}=0 \\
& z=-b, z=b: u=0, v=0, \omega=0, \frac{\partial p}{\partial z}=0
\end{aligned}
$$

With the governing Eqs. (1) (2) and boundary conditions, the analytic solutions can be easily.

derived by separation method:

flow velocity:

$$
u(y, z)=\frac{16 a^{2}}{\mu \pi^{3}}\left(-\frac{\partial p}{\partial x}\right) \times A_{m}
$$

flow rate:

$$
Q=\int_{-a}^{a} \int_{-b}^{b} u(y, z) d y d z=\frac{b a^{3}}{6 \mu}\left(-\frac{\partial p}{\partial x}\right) \times B_{m}
$$

where

$$
\begin{aligned}
& A_{m}=\sum_{m=1,3,5}^{\infty}(-1)^{(m-1) / 2}\left[1-\frac{\cosh \left(\frac{m \pi z}{2 a}\right)}{\cosh \left(\frac{m \pi b}{2 a}\right)} \frac{\cos \left(\frac{m \pi y}{2 a}\right)}{m^{3}}\right. \\
& B_{m}=\left[1-\frac{192 a}{\pi^{5} b} \sum_{m=1,3,5}^{\infty} \frac{\tanh (m \pi b / 2 a)}{m^{5}}\right]
\end{aligned}
$$

If aspect ratio of cross section $X=a / b$ is defined, the solution (3), (4) can be rewritten as

$$
Q=\frac{a^{3} I B L_{p}}{6 \mu L^{2}}\left[1-\frac{192}{\pi^{5}} X \sum \frac{\tanh \left(\frac{m \pi}{2 X}\right)}{m^{5}}\right]
$$

$\bar{V}($ average flow velocity $)=\frac{Q}{A}$

$$
=\frac{a B I L_{p}}{24 \mu L^{2}} X\left[1-\frac{192}{\pi^{5}} X \sum \frac{\tanh \left(\frac{m \pi}{2 X}\right)}{m^{5}}\right]
$$

Replacing the terms in square brackets of solution (6) as Y, the Eqs. (5) (6) will become as:

$$
\begin{aligned}
Q & =\frac{a^{3} I B L_{p}}{6 \mu L^{2}}\left(\frac{Y}{X}\right) \text { for width of duct } 2 \text { a fixed } \\
& =\frac{b^{3} I B L_{p}}{6 \mu L^{2}}\left(X^{2} Y\right) \text { for depth of duct } 2 \mathrm{~b} \text { fixed }
\end{aligned}
$$




$$
\begin{aligned}
\bar{V} & =\frac{a B I L_{p}}{24 \mu L^{2}} Y \text { for width of duct } 2 \mathrm{a} \text { fixed } \\
& =\frac{b B I L_{p}}{24 \mu L^{2}}(X Y) \text { for depth of duct } 2 \mathrm{~b} \text { fixed }
\end{aligned}
$$

where

$$
Y=X\left[1-\frac{192}{\pi^{5}} X \sum \frac{\tanh \left(\frac{m \pi}{2 X}\right)}{m^{5}}\right]
$$

\section{Flow circuit}

To describe the flow resistance in the channel and simplify the calculating procedure, the equivalent circuit which is analogous to the thermal network in heat conduction will be formulated by transforming Es. (7)

(8) as $I B L_{p}=Q * \frac{6 \mu L^{2}}{a^{3}}\left(\frac{X}{Y}\right)$ or $Q * \frac{6 \mu L^{2}}{b^{3}}\left(\frac{1}{X^{2} Y}\right)$ and the flow circuit can be set up as

The driving source of Lorentz force $I B L_{p}$ is similar to the voltage $\mathrm{V}$ in electric circuit and the flow resistance $\frac{6 \mu L^{2}}{a^{3}}\left(\frac{X}{Y}\right)$ or $\frac{6 \mu L^{2}}{b^{3}}\left(\frac{1}{X^{2} Y}\right)$ just behaves like the electric resistance $\mathrm{R}$.

\section{Experimental method}

A schematic view of the experiment channel is shown in Figure 2. The present set consists of six major portions, namely, liquid reservoir, a power supply, duct channel, glass piece, copper sheet and high density magnetic. To construct the duct channel whose dimensions are illustrated in Table 1, an acrylic resin board $\left(80 * 80 * 10 \mathrm{~mm}^{3}\right)$ was milled along its symmetric axis. Both sidewalls at the middle portion of channel were made of copper electrodes $\left(35 * 12 * 0.1 \mathrm{~mm}^{3}\right)$ which made the current across the channel possible. Orthogonal to the direction of current and test channel, the permanent magnets were positioned just above the glass piece, the copper sheet and below the acrylic resin board. In additions, ranging from voltage 3-12 $\mathrm{V}$ and current 0-3 A were also regulated respectively by a power supply (GPS-3030) with $1 \%$ output error in this study.

Before the working voltage is in action, the working medium first flows down from the reservoir to test

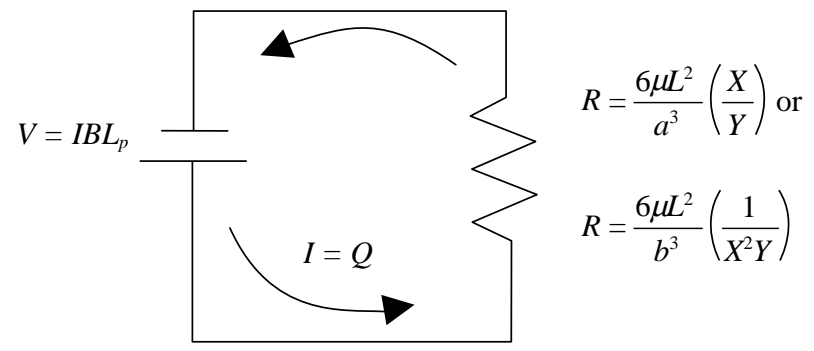

Fig. 1. MHD flow circuit.

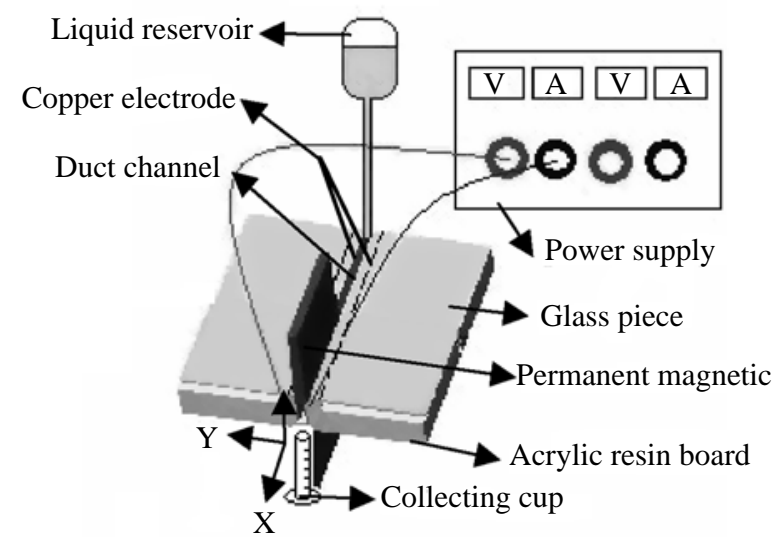

Fig. 2. Schematic drawing of MHD pump.

Table 1. Parameters for experimental apparatus

\begin{tabular}{ll}
\hline \multicolumn{1}{c}{ Parameter } & \multicolumn{1}{c}{ Value } \\
\hline Channel depth, $2 \mathrm{~b}(\mathrm{~m})$ & 0.007 \\
Channel width, $2 \mathrm{a}(\mathrm{m})$ & $0.003 \sim 0.02$ \\
Channel length, $L(\mathrm{~m})$ & 0.08 \\
Electrode length $L_{p}(\mathrm{~m})$ & 0.035 \\
Magnetic flux density, $B(\mathrm{~T})$ & 0.02 \\
Input current, $I(\mathrm{~A})$ & $0.1 \sim 1.3$ \\
Working medium: $(\mathrm{NaCl}$ solution$)$ & \\
Density, $\rho\left(\mathrm{kg} / \mathrm{m}^{3}\right)$ & 1058 \\
Conductivity, $\sigma(\mathrm{S} / \mathrm{m})$ & 1.5 \\
Viscosity, $\mu(\mathrm{Pas})$ & 0.0006 \\
\hline
\end{tabular}

channel till the steady state is reached and then the flow volume is colleted with measuring cup in 60s. Repeat the above process but with the application of working voltage, the Lorentz force which is interacted between the electric field and magnetic field begins to drive the working medium to flow through the test channel. After steady state is reached, the volume is also collected as above mentioned. The difference in both volume collected will determine the working capacity of Lorentz force. 


\section{RESULTS AND DISCUSSION}

\section{The effect of geometry}

To understand the pumping performance with dimensions of channel, the mean flow velocity versus with the width or depth of duct will be discussed respectively. From the solutions $(7) \sim(10)$, mean velocity and flow rate can be expressed as function $(\mathrm{X}, \mathrm{Y})$ where $\mathrm{X}$ is the ratio of width and depth in duct and $\mathrm{Y}$ is defined as average velocity factor which is also dependent with $\mathrm{X}$. To avoid the confusion on the definition of aspect ratio $X$ in both case, we redefine $X_{1}$ as the aspect ratio for fixed width with various depth of duct and $X_{2}$ as the aspect ratio for fixed depth with various widths. Although if $X_{1}$ equals $X_{2}$, it can't still predict the same size of aspect in both case.

First, the variation of depth with width 2 a fixed is concerned; the mean flow velocity in solution (9) shows a linear increase with the parameter $\mathrm{Y}$. That means the distribution of $\mathrm{Y}\left(X_{1}\right)$ can be used to predict mean flow velocity with $X_{1}$. Before we start to discuss, the interpretation of aspect ratio $X_{1}$ in real shape of duct is needed. As a larger $X_{1}$ is more than unity (which indicates the scalar depth is less than its width), a shallower duct will present. Otherwise, a deeper duct will be considered. In Figure 3, the value of $Y$ will gradually decrease as the $X_{1}$ increases from $1.0 \sim 4.0$. It tells the mean velocity will be further slower in the shallower duct due to the retarding of flow motion caused by the viscous effect at the small gap between top and bottom of the channel. In contrast to above, the $\mathrm{Y}$ will drastically drop from 0.42 to 0.09 as $X_{1}$ decreases from $1.0 \sim 0.1$ where the scalar depth is larger than its width. Since deeper duct presents as the $X_{1}$ decreases, the applied current density on the sidewalls will be further reduced; that will make the Lorentz force become too weak to drive the flow well. In Figure 3, another interesting motion is worthy mentioned that the maximum $\mathrm{Y}$ is observed at $X_{1}=1$; it indicates the maximum mean flow velocity will come out if the square cross section of duct is applied.

Although both factors arisen by lower current $\left(X_{1}\right.$ $<1.0)$ and higher viscous friction $\left(X_{1}>1.0\right)$ will slow down the mean flow motion and reduce the factor $Y$, the slope of curve $\left(X_{1}=0.1 \sim X_{1}=1.0\right)$ seems to be much greater than that in any other where; it implies the influence of current density is more active than that in viscous drag in this case.

The dependence of $X_{2}$ Y with $X_{2}$ was illustrated in Figure 4 which applies to the fixed depth $2 b$ with various widths in duct. Since the dependence of $X_{2} Y$ behaves a linear increase with mean flow velocity from solution (10), the distribution of $X_{2} Y$ will be used as an indicator for mean flow velocity with $X_{2}$. In this section, a small $X_{2}$ (less than unity) implies a narrower duct. Otherwise, a wider duct will be considered. From the shown in Figyre 4, the $X_{2} \mathrm{Y}$ presents an abrupt decrease from $0.4 \sim 0$ as the $X_{2}$ decreases from $1 \sim 0$. It tells that the mean flow velocity will be further reduced due to the viscous drag of sidewalls deeply influences the flow motion in the narrower duct. As aspect ratio increases from $1 \sim 4$, the dependence $X_{2} \mathrm{Y}$ grows gradually to a stable value 0.75 where the frictional effect of sidewalls has lost its influence and the flow velocity will approach to a constant value in the wider duct.

The analytic results for the average flow velocity with various dimensions of channel under the applied current $0.7 \mathrm{~A}$ and magnetic flux density $0.02 \mathrm{~T}$ are shown in Figure 5. To square section of the channel, the flow velocity $0.00098 \mathrm{~m} / \mathrm{s}$, flow rate $0.01 \mathrm{ml} / \mathrm{s}$ are presented at $1 \mathrm{~mm}^{2}$ square cross section and then $0.039 \mathrm{~m} / \mathrm{s}, 626$ $\mathrm{ml} / \mathrm{s}$ is for $40^{2} \mathrm{~mm}^{2}$ square section. With above description, the average velocity nearly exhibits a linear increase with width but the flow rate approximately predicts a increase with the cubic of width. In contrast to the square dimensions of channel, the average velocity will behave like the distributions in Figure 3 and

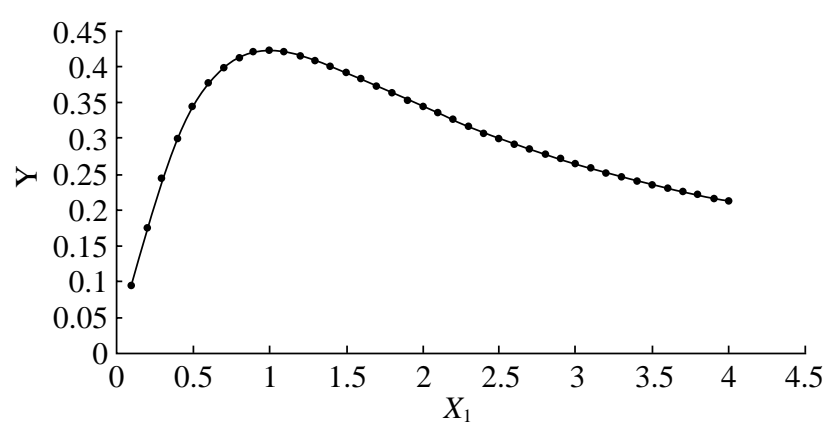

Fig. 3. Average flow velocity factor $Y$ vs aspect ratio $X_{1}$ while the width of duct is fixed.

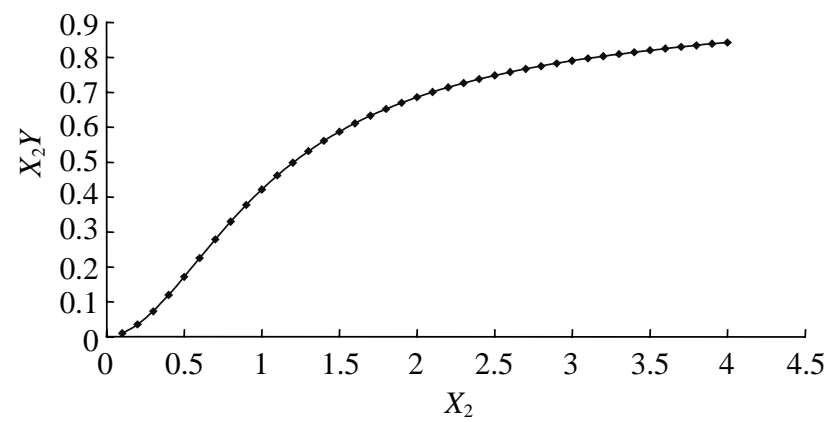

Fig. 4. Average flow velocity factor $X_{2} Y$ vs aspect ratio $X$ while the depth of duct is fixed. 


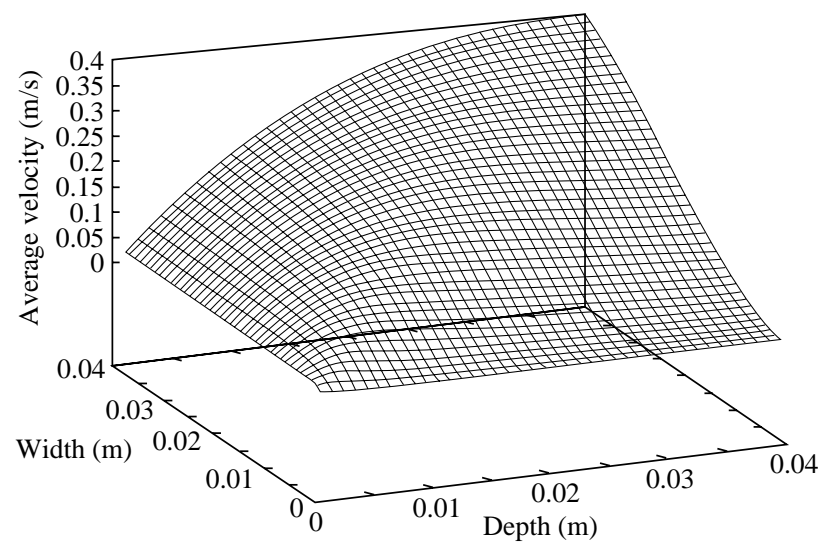

Fig. 5. Average flow velocity vs dimensions of duct channel under the input current 0.7A, magnetic flux density $0.02 \mathrm{~T}$.

Figure 4 which are both of the characteristic curves in Figure 5.

Before we start next discussion, four distinct aspect shapes will be classified from the above analysis. Deeper duct $\left(X_{1}<1\right)$ or shallower duct $\left(X_{1}>1\right)$ is considered in the case with width fixed and the narrower duct $\left(X_{2}<1\right)$ or wider duct $\left(X_{2}>1\right)$ is defined in the fixed depth case. Comparison form the Figure $3 \sim$ Figure 5, mean flow velocity in Figure 3 will be more than that in Figure 4 when aspect ratio is less than unity; but the reversed result will come out while the ratio is more than unity.

\section{Flow resistance}

To set up the flow circuit in Figure 1 which is similar to the electric circuit, the analytic solutions in Eq. (7) $\sim(8)$ should be rearranged. Lorentz force $I B L_{p}$ is performed as the voltage in the electric circuit and the flow resistance $\mathrm{R}$ expressed as the $\mathrm{X} / \mathrm{Y}$ or $1 /\left(\mathrm{XY}^{2}\right)$ according to individual case is only dependent with the aspect of duct.

As the fixed width with different depths are concerned, flow resistance $\mathrm{R}$ has linear relationship with $X_{1} / Y$ from the solution (7) and the factor of $X_{1} / Y$ in Figure 6 remains about $1 \sim 2$ at the smaller $X_{1}(0.1 \sim 1)$ where predicts a deeper duct applied. In contrast to this, the factor will begin to quickly climb from $2 \sim 20$ while $X_{1}$ varies from $1 \sim 4$ where a shallower duct is in use.

According to above description, a larger $\mathrm{R}$ will exist at the shallower channel where the viscous drag between the upper and bottom has a strong influence on flow motion. But for deeper channel, the viscous drag has been gradually reduced as the depth of duct increases; that results in a lower resistance $\mathrm{R}$ in the channel.

As the fixed depth with various widths are discussed, flow resistance $\mathrm{R}$ has linear relationship with $1 /\left(\mathrm{Y} \mathrm{X}_{2}^{2}\right)$ from the solution (8). The dependence of $1 /\left(\mathrm{Y} X_{2}^{2}\right)$ in Figure 7 shows a drastic decay from 1000 $\sim 5$ as the aspect ratio $X_{2}$ increases from $0.1 \sim 1$ (narrower duct) and then gradually decreases to a stable value 0.5 while $X_{2}$ is extended to 4 (wider duct). That indicates the viscous drag between the sidewalls in the narrower duct $\left(X_{2}<1\right)$ will become dominated to produce a higher flow resistance $\mathrm{R}$ in the channel. On the other hand, the $\mathrm{R}$ will keep a lower value in the wider duct $\left(X_{2}>1\right)$ where the frictional effect of sidewalls has gradually lost its influence on flow motion .

Comparing the Figure 6 and Figure 7, an interesting distribution in resistance factor or $\mathrm{R}$ presents. The factor will quickly increase with the aspect ratio in the case with the width fixed in Figure 6, but an opposite tendency comes out in the case with the depth fixed in Figure 7. Although the more flow rate with lower flow resistance for deeper case $\left(X_{1}<1\right)$ in Figure 6 and the wider case $\left(X_{2}>1\right)$ in Figure 7 are proposed, the needed power to maintain the same Lorentz force will be much increased because the current density on the electrode plate or the working current in the channel becomes less. A solution with unit aspect ratio (square cross

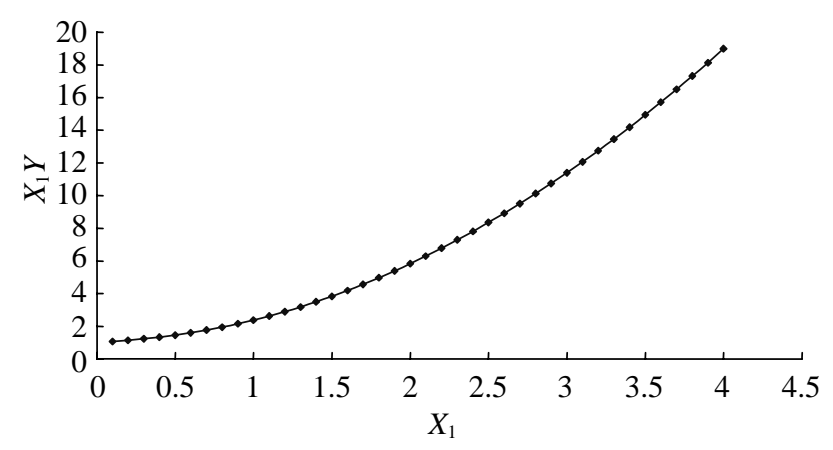

Fig. 6. The flow resistance factor $X_{1} Y$ vs the aspect ratios $X_{1}$ while the width of duct is fixed.

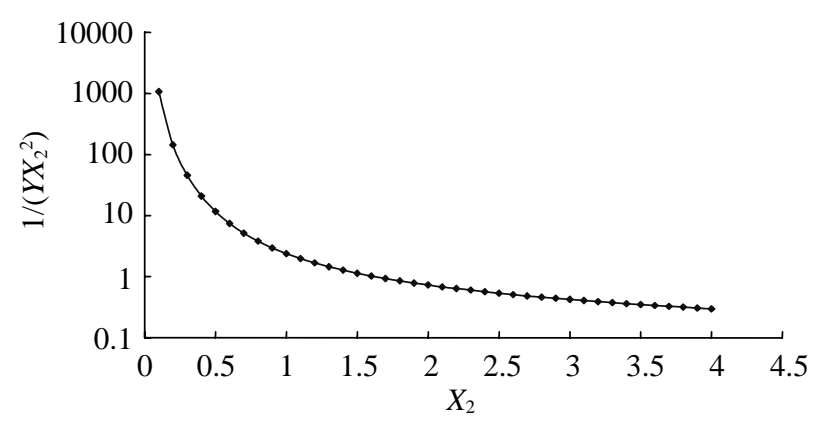

Fig. 7. The flow resistance factor $\left(Y X_{2}^{2}\right)^{-1}$ vs the aspect ratios $X_{2}$ while the depth of duct is fixed. 
section) will be found to make the dependent value in Figure 6 and in Figure 7 equal. Besides it meets the relative smaller resistance factor about 5.0 for both cases, it is also the solution for general pressure-driven flow with minimum viscous drag.

\section{Comparison with the analytic solution and experiment result}

To verify the correct of the analytic solution, the experiments made by varying width of duct were shown in Figure 8. Both results were proven to be well agreement with each other and their maximum relative error was not more than $10 \%$ at the width $1.5 * 10^{-2} \mathrm{~m}$ corresponding to flow rate about $13 \mathrm{ml} / \mathrm{s}$.If the width is less than $2 \mathrm{~mm}$, flow rate measured will not discussed here because small bubbles produced during experimental process will easily retard the flow motion in the narrower channel.

In Figure 9, the analytic solution $(0.003 \mathrm{~m} / \mathrm{s} \sim$ $0.039 \mathrm{~m} / \mathrm{s})$ and experiment result $(0.003 \mathrm{~m} / \mathrm{s} \sim 0.034$ $\mathrm{m} / \mathrm{s}$ ) for average flow velocity present a linear increase with the applied current $(0.1 \mathrm{~A} \sim 1.3 \mathrm{~A})$. When compared with above data, their deviations little increase with working current; but not more than $15 \%$ during the current varies from $0.1 \mathrm{~A}$ to $1.3 \mathrm{~A}$. As is the increase of the applied current, bubbles' growth in aqueous solutions becomes to affect the flow motion; it will make the analytic solution begin to departure form the experimental result.

\section{CONCLUSIONS}

Summary from previous discussion, several important conclusions may be made as follows:

1. If a steady, fully developed MHD flow problem is discussed, the assumption of uniform distribution for Lorentz force along the flow channel will make the analytic solution possible

2. The set up of flow circuit similar to the thermal network in heat conduction provides an easy way to evaluate the flow resistance in flow channel.

3 . With different aspect ratio, the shape of duct will be classified as shallower, deeper, narrower and wider case. That gives a general discussion with more convenient and efficient in this study.

4. Though a lower flow resistance presents as the deeper duct is applied, the current density on the sidewalls will be also further reduced simultaneously; that brings about a gradual decrease for flow rate. On the other hand, an extra voltage is needed to keep the constant current for the wider duct with lower flow resistance.

5 . As deeper duct $x \rightarrow 0$, flow rate measured will be usually unstable due to the retarding of smaller bubbles

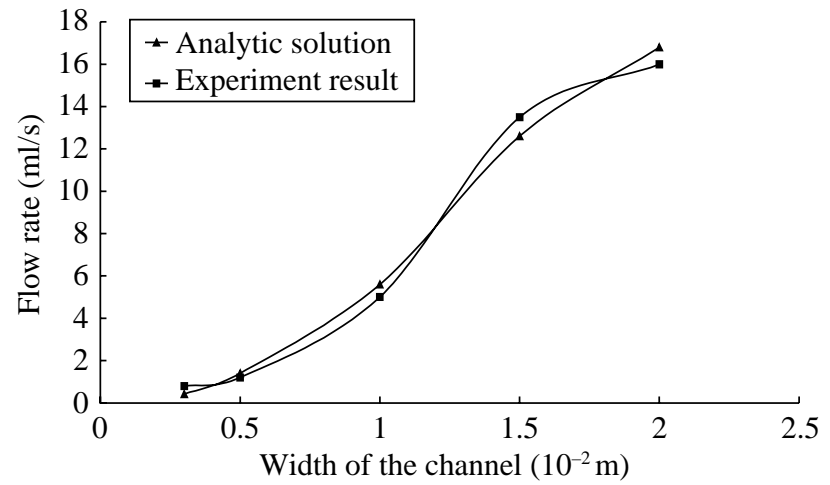

Fig. 8. Analytic flow rate vs experiment result while the input current is $0.7 \mathrm{~A}$ and the magnetic density is $0.02 \mathrm{~T}$.

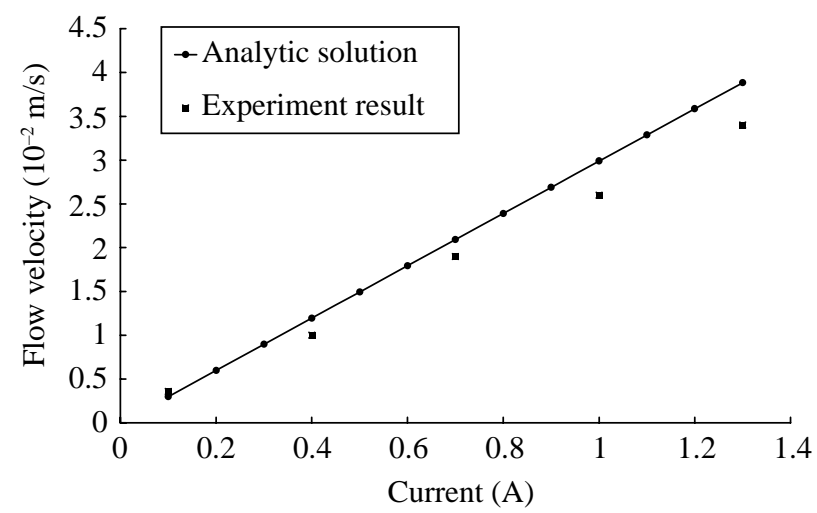

Fig. 9. Comparison the flow velocity between analytic solution and experiment result with applied current.

in the flow channel. On the other hand, the larger bubbles produced under higher applied current will raise some discrepancy between the analytic solution and experimental results.

\section{REFERENCES}

1. Geng, X., Yuan, H., Oguz, H.N., and Prosperetti, A., "Bubble-Based Micro Pump for Electrically Conducting Liquids," Journal of Micromechanics and Micro Engineering, Vol. 11, pp. 270-276 (2001).

2. Ho, J.E., "The Effect of Bubbles in MHD Micropump," Proceeding of the $22^{\text {nd }}$ National Conference on the Chinese Society of Mechanical Engineers, pp. 903-910 (2005).

3. Ho, J.E., "The Optima Geometry of Duct Channel in MHD Pump" Advances in Micro and Nano Technology pp. 96-99 (2006).

4. Huang, L., Wang, W-S, Murphy, M.C., Lian, K., and Ling, Z.G., "2000 LIGA Fabrication and Test of a Dc Type Magnete-Hydrodynamic (MHD) Micro-Pump," 
Microsystem Technologies, Vol. 6, pp. 235-240 (2000).

5. Jang, J. and Lee, S.S., "Theoretical and Experimental Study of MHD (Magne to Hydro Dynamic) Micropump," Sensors and Actuators A, Vol. 80, pp. 84-89 (2000).

6. Verardi, S.L.L., Cardoso, J.R., and Costa, M.C., "ThreeDimensional Finite Element Analysis of MHD Duct
Flow by the Penalty Function Formulation" IEEE Transactions on Magnetics, Vol. 37, No. 5, pp. 33843387 (2001).

7. Wang, P.J., Chang, C.Y., and Chang, M.L., "Simulation of Two-Dimensional Fully Developed Laminar Flow for A Magneto-Hydrodynamic (MHD) Pump," Biosensors and Bioelectronics, Vol. 20, pp. 115-121 (2004). 